\title{
Variation in Antioxidant Activity and Polyphenol Content in Tomato Stems and Leaves
}

\author{
Xiang-Min Piao, Eun-Kyu Jang, Jong-Wook Chung, Gi-An Lee, Ho-Sun Lee, Jung-Sook Sung, \\ Young-Ah Jeon, Jung-Ro Lee, Yeon-Gyu Kim, Sok-Young Lee* \\ National Agrobiodiversity Center, NAAS, RDA, Suwon, 441-853, Republic of Korea
}

\begin{abstract}
Tomato was considered as one of the most widely cultivated vegetable crops in the world. Tomato plant has high antioxidant capacity which can be attributed to the high levels of carotenoids, phenols, vitamins $\mathrm{C}$ and E. However, most of tomato plants have been discarded as waste after fruit harvesting. In order to identify genetic resources with high antioxidant level for use in food or as feed additives, we investigated the ABTS, DPPH antioxidant activity and polyphenol content in tomato leaves and stems. A total of 112 tomato accessions were classified into three groups by latitude of their collected countries: $30^{\circ} \sim 60^{\circ}$ North (50 accessions), $0^{\circ} \sim 30^{\circ}$ North (40 accessions), and $0^{\circ} \sim 30^{\circ}$ South (22 accessions). Stem and leaf extracts showed wide variation in ABTS antioxidant activity ranging from $1.6 \pm 1.0$ to $48.4 \pm 6.1 \mu \mathrm{g}$ Trolox $\mathrm{mg}^{-1} \mathrm{dw}$. The antioxidant activity of DPPH was in the range of $6.3 \pm 0.2$ to 40.0

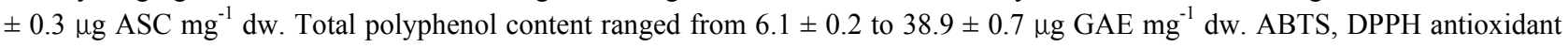
activities and polyphenol content in accessions from $30^{\circ} \sim 60^{\circ} \mathrm{N}$ latitude were significantly higher $(\mathrm{P}<0.05)$ than those from $0^{\circ} \sim 30^{\circ} \mathrm{N}$ latitude. ABTS values showed a significant positive correlation $\left(\mathrm{r}=0.700^{* *}\right)$ with DPPH activity. IT100506 (KOR) and 702959 (UKR) were recommended as potential sources of natural antioxidants due to their highest antioxidant activity among accessions. This study will provide valuable information for tomato breeders in developing and producing functional food or feed additives resources.
\end{abstract}

Keywords Antioxidant activity, Polyphenol, Tomato, Stems and leaves

\section{INTRODUCTION}

Tomato (Solanum lycopersicum L.) was considered as one of the most widely cultivated vegetable crops in the world (Hanson et al. 2004; Borguini and Torres 2009). Tomato plant has high antioxidant capacity which can be attributed to the high levels of carotenoids, phenols, vitamins $\mathrm{C}$ and $\mathrm{E}$ (Kotkov et al. 2009). Antioxidants act to both reduce the content of toxic components in foods and to supply the human body with exogenous antioxidant (Block and Langseth 1994). Antioxidant capacity depends on the tomato variety, environmental growth conditions, production techniques used, and post-harvest storage conditions (Dumas et al. 2003). Low temperatures and northern latitudes have been reported to increase the amounts of antioxidants in berries and walnuts (Åkerstöm et al. 2010; Ghasemi et al. 2011). Methods using the stable 2,2'-azino-bis (3-ethylbenzothiazoline-6-sulphonic acid) (ABTS) or 1,1diphenyl-2-picryl-hydrazil (DPPH) radicals are used widely to evaluate the free radical scavenging ability of antioxidant substances (Nabavi et al. 2009). Both methods are characterized by excellent reproducibility under certain assay conditions, but also show significant differences in their responses to antioxidants. The $\mathrm{ABTS}^{+}$can be dissolved in aqueous and organic media due to the hydrophilic and lipophilic nature of the compounds present in samples. In contrast, DPPH is soluble only in organic media, especially ethanol, this being an important limitation when interpreting the role of hydrophilic antioxidants (Arnao 2000).

Previous studies revealed that ethanol extracts of tomato, eggplant, and sweet potato leaves have higher antioxidant activity, phenolic components and flavonol content than their fruits (Zornoza and Esteban 1984; Truong et al. 2007; Jung et al. 2011; Munir et al. 2012). Hence, these leaves

Received October 11, 2013; Revised October 28, 2013; Accepted October 29, 2013; Published December 31, 2013

*Corresponding author Sok-Young Lee, 1sy007@korea.kr, Tel: +82-31-299-1821, Fax: +82-31-294-6029 
represent a potential source of natural antioxidants. However, during the harvest period, $95-98 \%$ of tomato leaves and stems are discarded while the remaining $2-5 \%$ is used as animal food (Mcgee 2009). The reason is that foliage of the tomato plant has long been considered potentially toxic because of the alkaloid tomatine. However, levels of tomatine in leaves and stems are generally too small to be dangerous unless large amounts are consumed (Barceloux 2009). The recent research found that tomatine binds to cholesterol molecules in the digestive system. In fact, ingesting the leaves can lower the levels of undesirable LDL cholesterol in humans and animals (Mcgee 2009). Research into the antioxidant capacity of tomato stems and leaves compared to fruits is limited, and little research has aimed to determine the influence of collection latitudes on the antioxidant activity in tomato stems and leaves. In this study, the DPPH and ABTS activities and polyphenol contents of the leaves and stems of 112 tomato accessions originating from 18 countries were investigated to determine the effects of collection latitudes on antioxidant activity and polyphenol content, and also to identify high antioxidant activity tomato germplasm which can be used as a source of feed additive.

\section{MATERIALS AND METHODS}

\section{Materials}

One hundred and twelve tomato accessions were obtained from the National Agro-biodiversity Center. All accessions collected from 18 countries were classified into three groups by latitude of their origins: $30^{\circ} \sim 60^{\circ}$ North $(n=50)$, $0^{\circ} \sim 30^{\circ}$ North $(\mathrm{n}=40)$, and $0^{\circ} \sim 30^{\circ}$ South $(\mathrm{n}=22)$. The $30^{\circ} \sim 60^{\circ} \mathrm{N}$ latitude area is composed of nine countries: NLD, DEU, HUN, BGR, UKR, ARM, UZB, KOR, and JPN. The $0^{\circ} \sim 30^{\circ} \mathrm{N}$ latitude area included six countries: ETH, IND, TWN, PHL, HND, and CUB. The $0^{\circ} \sim 30^{\circ}$ $S$ latitude area is composed of three countries: ZWE, PER, and BRA (Fig. 1, Table 1). The accessions were grown in an experimental field in Suwon during April 2012. Plant spacing was $50 \mathrm{~cm}$ between rows and 40 $\mathrm{cm}$ between plants.

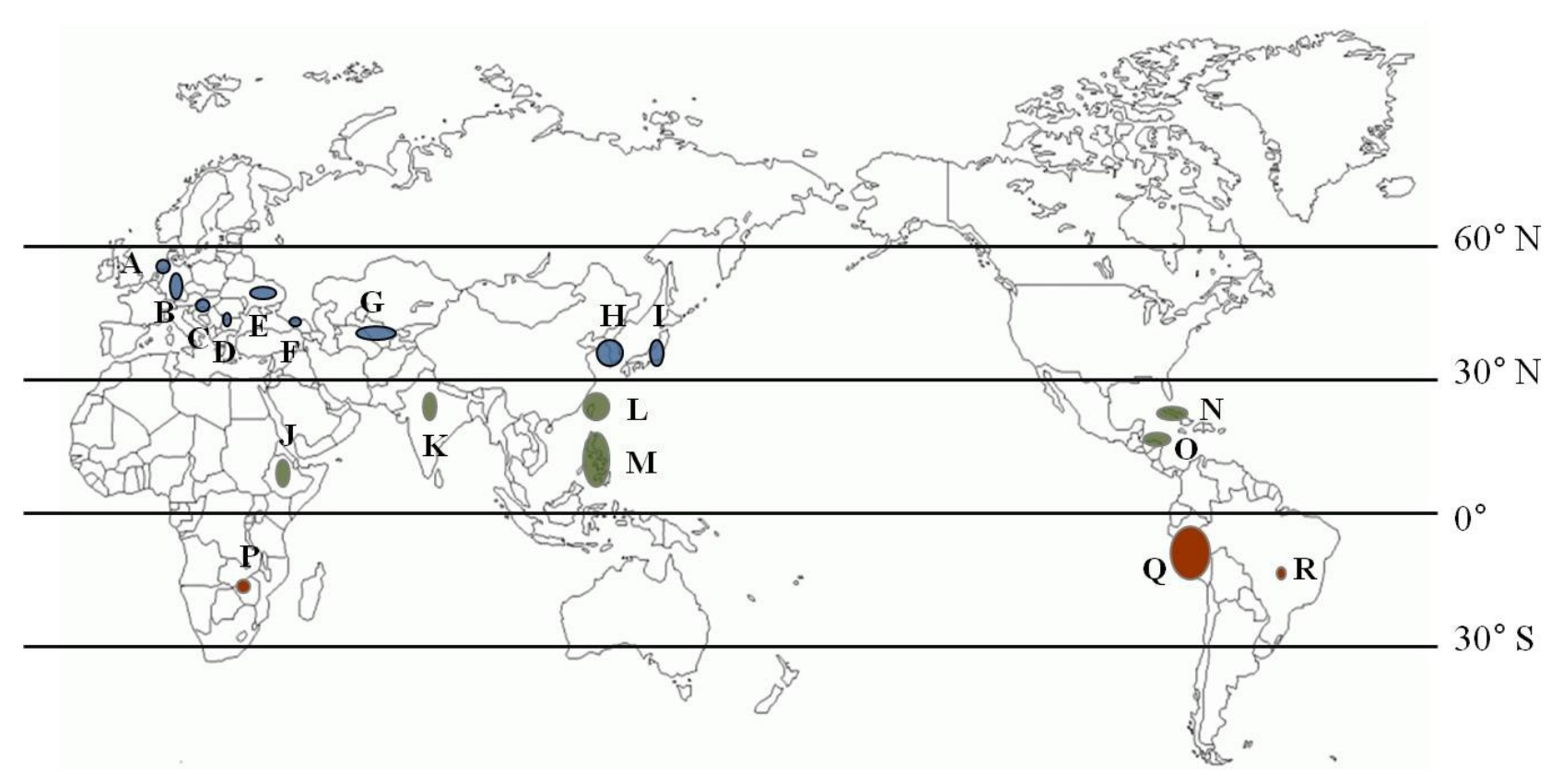

Fig. 1. Distribution of 112 tomato accessions according to country of origin.

A: $\operatorname{NLD}(n=4), B: \operatorname{DEU}(n=2), C: \operatorname{HUN}(n=1), D: \operatorname{BGR}(n=2), E: \operatorname{UKR}(n=3), F: \operatorname{ARM}(n=2), G: \operatorname{UZB}(n=10), H$ : $\operatorname{KOR}(n=22), I: \operatorname{JPN}(n=3), J: \operatorname{ETH}(n=1), K: \operatorname{IND}(n=2), \operatorname{L}: \operatorname{TWN}(n=10), \operatorname{M}: \operatorname{PHL}(n=23), N: \operatorname{HND}(n=1), O$ : $\operatorname{CUB}(\mathrm{n}=1), \operatorname{P}: \operatorname{ZWE}(\mathrm{n}=1), \mathrm{Q}: \operatorname{PER}(\mathrm{n}=20), \mathrm{R}: \operatorname{BRA}(\mathrm{n}=1)$ 
Table 1. NAC registration numbers and origins of 112 tomato accessions investigated in this study.

\begin{tabular}{|c|c|c|c|c|c|c|c|}
\hline $\begin{array}{l}\text { NAC registration } \\
\text { number }\end{array}$ & $\begin{array}{c}\text { Country of } \\
\text { origin }\end{array}$ & $\begin{array}{c}\text { NAC registration } \\
\text { number }\end{array}$ & $\begin{array}{c}\text { Country of } \\
\text { origin }\end{array}$ & $\begin{array}{c}\text { NAC } \\
\text { registration } \\
\text { number }\end{array}$ & $\begin{array}{c}\text { Country of } \\
\text { origin }\end{array}$ & $\begin{array}{l}\text { NAC registration } \\
\text { number }\end{array}$ & $\begin{array}{c}\text { Country of } \\
\text { origin }\end{array}$ \\
\hline 1. IT203258 & ARM & 29. K012913 & KOR & 57. IT173888 & PER & 85. IT116970 & PHL \\
\hline 2. IT203272 & ARM & 30. K012916 & KOR & 58. IT173901 & PER & 86. IT201662 & PHL \\
\hline 3. IT199436 & BGR & 31. K012920 & KOR & 59. IT173955 & PER & 87. IT201664 & PHL \\
\hline 4. K047416 & BGR & 32. K012924 & KOR & 60. IT174011 & PER & 88. IT116989 & TWN \\
\hline 5. K047418 & BRA & 33. K012934 & KOR & 61. IT203416 & PER & 89. K000872 & TWN \\
\hline 6. IT199463 & CUB & 34. K012970 & KOR & 62. K057603 & PER & 90. K000893 & TWN \\
\hline 7. 803106 & DEU & 35. K047488 & KOR & 63. IT116894 & PHL & 91. K177639 & TWN \\
\hline 8. K004846 & DEU & 36. K047491 & KOR & 64. IT116895 & PHL & 92. K177641 & TWN \\
\hline 9.805811 & ETH & 37. K047500 & KOR & 65. IT116897 & PHL & 93. K177642 & TWN \\
\hline 10. K047588 & HND & 38. K047503 & KOR & 66. IT116898 & PHL & 94. K177644 & TWN \\
\hline 11. K020958 & HUN & 39. K004905 & NLD & 67. IT116899 & PHL & 95. K177645 & TWN \\
\hline 12. IT136595 & IND & 40. K020956 & NLD & 68. IT116901 & PHL & 96. K177646 & TWN \\
\hline 13. IT203407 & IND & 41. K019075 & NLD & 69. IT116902 & PHL & 97. K177647 & TWN \\
\hline 14. IT186735 & JPN & 42. K019076 & NLD & 70. IT116903 & PHL & 98. IT203255 & UKR \\
\hline 15. IT186736 & JPN & 43. IT119947 & PER & 71. IT116904 & PHL & 99. 702959 & UKR \\
\hline 16. IT100506 & KOR & 44. IT119953 & PER & 72. IT116905 & PHL & 100. 702977 & UKR \\
\hline 17. K012777 & KOR & 45. IT173727 & PER & 73. IT116907 & PHL & 101. K020933 & UKR \\
\hline 18. K012781 & KOR & 46. IT 173730 & PER & 74. IT116908 & PHL & 102. IT199433 & UZB \\
\hline 19. K012793 & KOR & 47. IT173733 & PER & 75. IT116910 & PHL & 103. IT203240 & UZB \\
\hline 20. K012798 & KOR & 48. IT173742 & PER & 76. IT116912 & PHL & 104. IT203248 & UZB \\
\hline 21. K012807 & KOR & 49. IT173749 & PER & 77. IT116913 & PHL & 105. IT203252 & UZB \\
\hline 22. K012827 & KOR & 50. IT173750 & PER & 78. IT116914 & PHL & 106. IT203253 & UZB \\
\hline 23. K012841 & KOR & 51. IT173758 & PER & 79. IT116916 & PHL & 107. IT203254 & UZB \\
\hline 24. K012851 & KOR & 52. IT173759 & PER & 80. IT116918 & PHL & 108. IT203261 & UZB \\
\hline 25. K012859 & KOR & 53. IT173760 & PER & 81. IT116919 & PHL & 109. 805835 & UZB \\
\hline 26. K012888 & KOR & 54. IT173772 & PER & 82. IT116955 & PHL & 110. 908870 & UZB \\
\hline 27. K012893 & KOR & 55. IT173804 & PER & 83. IT116957 & PHL & 111. K014621 & UZB \\
\hline 28. K012904 & KOR & 56. IT173812 & PER & 84. IT116961 & PHL & 112.805750 & ZWE \\
\hline
\end{tabular}

\section{Methods}

\section{Chemicals}

1,1-diphenyl-2-picryl-hydrazil (DPPH•), L-ascorbic acid, 2,2'-azino-bis (3-ethylbenzothiazoline-6-sulphonic acid) (ABTS•), 6-hydroxy-2,5,7,8-tetramethylchroman-2-carboxylic acid (Trolox), Folin-Ciocalteu reagent, and gallic acid were obtained from Sigma-Aldrich (USA). All other reagents were of analytical grade.

\section{Sample preparation}

Crude extracts were produced using $7 \mathrm{~g}$ of oven-dried tomato stems and leaves using an ASE-200 (Dionex) extractor. Extractions were performed in $40-\mathrm{ml} 75 \%$ ethanol under nitrogen gas at a pressure of $1,500 \mathrm{psi}$ and at $70^{\circ} \mathrm{C}$. Extracted samples were dried using a Genevac HT-4X vacuum concentrator. 


\section{DPPH assay}

The free radical scavenging activity of the extracts was assessed by the DPPH $\bullet$ method proposed by Lee and Lee (2004), with slight modification. DPPH solution $(150 \mu \mathrm{l} ; 150 \mu \mathrm{M}$, in anhydrous ethanol) was added to $100 \mu \mathrm{l}$ of sample solution. The mixture was shaken vigorously and left to stand at $25^{\circ} \mathrm{C}$ in the dark for 30 min. Absorbance at $517 \mathrm{~nm}$ was then measured in a spectrophotometer. DPPH free radical scavenging activity was calculated using the following equation:

$$
\begin{aligned}
& \text { DPPH・ scavenging effect }(\%)= \\
& {[1-(\mathrm{A} 0-\mathrm{A} 1) /(\mathrm{A} 2-\mathrm{A} 3)] \times 100,}
\end{aligned}
$$

where $\mathrm{A} 0$ is the absorbance of the sample, A1 is the absorbance of the sample blank, A2 is the absorbance of the control, and A3 is the absorbance of the control blank. Finally, the radical scavenging effect was expressed as $\mu \mathrm{g}$ L-ascorbic acid equivalent antioxidant capacity (ASC) per 1-mg dried extract ( $\mu \mathrm{g}$ ASC $\mathrm{mg}^{-1} \mathrm{dw}$ ).

\section{ABTS assay}

ABTS radical scavenging activity was estimated using the method of Re et al. (1999) with some modifications. ABTS radical cation was generated by adding $7 \mathrm{mM}$ ABTS to $2.45 \mathrm{mM}$ potassium persulphate followed by overnight incubation of the mixture in the dark at room temperature. The ABTS radical cation solution was diluted with methanol to obtain an absorbance of $0.7 \pm$ 0.02 at $735 \mathrm{~nm}$. Diluted ABTS radical cation solution (190 $\mu \mathrm{l})$ was added to $10 \mu \mathrm{L}$ of sample solution. After $6 \mathrm{~min}$, absorbance at $735 \mathrm{~nm}$ was determined using a spectrophotometer. The capability to scavenge the ABTS radical was calculated using the following equation:

$$
\begin{aligned}
& \text { ABTS scavenging effect }(\%)= \\
& {[1-(\mathrm{A} 0-\mathrm{A} 1) /(\mathrm{A} 2-\mathrm{A} 3)] \times 100,}
\end{aligned}
$$

where A0 is the absorbance of the sample, A1 is the absorbance of the sample blank, A2 is the absorbance of the control, and A3 is the absorbance of the control blank. The free radical-scavenging effect of each sample was reported as the Trolox equivalent antioxidant activity obtained by comparing the changes in absorbance at 735 $\mathrm{nm}$ in reaction mixtures containing a sample tomato extract or a Trolox equivalent.

\section{Polyphenol assay}

Total polyphenol content was measured using the modified Folin-Ciocalteu method (Waterhouse 2002). Folin-Ciocalteu reagent $(100 \mu \mathrm{l})$ was added to $100 \mu \mathrm{l}$ of sample solution and allowed to react at room temperature for $3 \mathrm{~min}$. After addition of $100 \mu \mathrm{l}$ of $2 \%$ sodium carbonate, the mixture was incubated at room temperature for $30 \mathrm{~min}$. Absorbance was measured at $750 \mathrm{~nm}$ using an ELISA reader with distilled water as a blank. Total phenolic content was reported as milligrams of gallic acid equivalents (GAE) per gram dried weight sample ( $\mu \mathrm{g}$ GAE $\mathrm{mg}^{-1}$ $\mathrm{dw})$.

\section{Statistical analysis}

Each sample was analyzed in triplicate and data were reported as means. Duncan's multiple range test (DMRT) were carried out to test any significant differences among tomato accessions collected from different latitudes by the SAS program (Software version 9.1, SAS Institute Inc.). Correlation coefficients were calculated to describe the relationship between DPPH and ABTS activity.

\section{RESULTS}

ABTS, DPPH antioxidant activity and polyphenol content in stem and leaf extracts of 112 tomato accessions were investigated to identify genetic resources with high antioxidant level for use in food or as feed additives. ABTS antioxidant activity showed wide variation from $1.6 \pm 1.0 \mu \mathrm{g}$ Trolox $\mathrm{mg}^{-1} \mathrm{dw}$ (IT116898 from PHL) to $48.4 \pm 6.1 \mu \mathrm{g}$ Trolox $\mathrm{mg}^{-1} \mathrm{dw}$ (IT100506 from KOR). DPPH antioxidant activity was found to be in the range $6.3 \pm 0.2 \mu \mathrm{g} \mathrm{ASC} \mathrm{mg}{ }^{-1}$ $\mathrm{dw}$ (IT116898) to $40.0 \pm 0.3 \mu \mathrm{g}$ ASC $\mathrm{mg}^{-1} \mathrm{dw}$ (702959 from UKR). Total polyphenol content ranged from 6.1 $\pm 0.2 \mu \mathrm{g} \mathrm{GAE} \mathrm{mg}^{-1} \mathrm{dw}$ (IT116898) to $38.9 \pm 0.7 \mu \mathrm{g}$ GAE $\mathrm{mg}^{-1} \mathrm{dw}$ (K12913 from KOR) in extracts of the stems and leaves of various tomato accessions (Table 2).

Duncan's multiple range test indicated that the ABTS, 
DPPH antioxidant activities and total polyphenol content in accessions from $30^{\circ} \sim 60^{\circ} \mathrm{N}$ latitude $\left(33.4 \mathrm{Trolox} \mathrm{mg}^{-1}\right.$

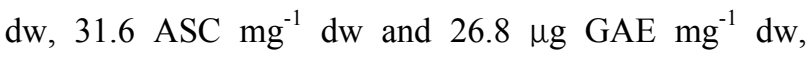
respectively) were significantly higher $(\mathrm{P}<0.05)$ than those from $0^{\circ} \sim 30^{\circ} \mathrm{N}$ latitude $\left(27.3\right.$ Trolox $\mathrm{mg}^{-1} \mathrm{dw}$, 26.4 $\mathrm{ASC} \mathrm{mg}^{-1} \mathrm{dw}$ and $23.2 \mu \mathrm{g} \mathrm{GAE} \mathrm{mg}^{-1} \mathrm{dw}$, respectively) (Table 3). It is considered that low latitude or high temperature of the geographical origins may lead to the low antioxidant activity and phenolic compounds accumulation. It is reported that the cultivated tomato is native to the Peru-Ecaudor area, and spread throughout the world following the Spanish colonization of the Americas (Pinela et al. 2012). In this study, DPPH antioxidant activity and total polyphenol content in accessions from Peru and Brazil $\left(0^{\circ} \sim 30^{\circ} \mathrm{S}\right.$ latitude $)$ were found to be significantly higher $(\mathrm{P}<0.05)$ than those from $0^{\circ} \sim 30^{\circ} \mathrm{N}$ latitude (Table 3).

The ABTS and DPPH activities showed skew-normal distributions (Figs. 2, 3). ABTS activity in two accessions from $30^{\circ} \sim 60^{\circ} \mathrm{N}$ latitude were more than $45 \mu \mathrm{g}$ Trolox $\mathrm{mg}^{-1}$ while in one accession from $0^{\circ} \sim 30^{\circ} \mathrm{N}$ latitude was less than $5 \mu \mathrm{g}$ Trolox $\mathrm{mg}^{-1} .84 \%$ of accessions from $30^{\circ}$

Table 2. ABTS and DPPH antioxidant activities and polyphenol contents of stem and leaf extracts of 112 tomato accessions.

\begin{tabular}{|c|c|c|c|c|c|c|c|c|c|c|c|c|c|c|c|}
\hline No. & $\mathrm{ABTS}^{\mathrm{z})}$ & $\mathrm{DPPH}^{\mathrm{y})}$ & Polyphenol ${ }^{\mathrm{x})}$ & No. & ABTS & DPPH & Polyphenol & No. & ABTS & DPPH & Polyphenol & No. & ABTS & DPPH & Polyphenol \\
\hline 1 & $25.5 \pm 3.9$ & $25.1 \pm 2.1$ & $3.2 \pm 1.0$ & 29 & $14.4 \pm 0.1$ & $21.9 \pm 0.1$ & & 57 & $23.3 \pm 1.6$ & $29 \pm 0.2$ & & 85 & & & \\
\hline 2 & $29.8 \pm 3.8$ & $17.8 \pm 1.8$ & $22.1 \pm 2.0$ & 30 & $36.7 \pm 0.9$ & $36.1 \pm 0.8$ & $28.7 \pm 4.3$ & 58 & $28.4 \pm 0.7$ & $32.5 \pm 0.5$ & $31.4 \pm 1.0$ & 86 & $36 \pm 2.7$ & $23.0 \pm 2.2$ & $17.1 \pm 0.5$ \\
\hline 3 & $27.5 \pm 2.1$ & $20.9 \pm 3.7$ & $21.9 \pm 0.5$ & 31 & $21.5 \pm 4.5$ & $7.3 \pm 0.3$ & $7.8 \pm 0.2$ & 59 & $22.6 \pm 1.2$ & $21.7 \pm 2.2$ & $34.0 \pm 1.4$ & 87 & $43.5 \pm 6.4$ & $30.2 \pm 1.7$ & $26.7 \pm 2.4$ \\
\hline 4 & $37.5 \pm 0.8$ & $39.9 \pm 0.2$ & $29.6 \pm 1.1$ & 32 & $33.1 \pm 1.5$ & $28.6 \pm 0.5$ & $30.2 \pm 1.1$ & 60 & $24.7 \pm 0.3$ & $29.6 \pm 0.5$ & $31.9 \pm 1.8$ & 88 & $19.2 \pm 1.8$ & $21.5 \pm 0.4$ & $16.2 \pm 0.9$ \\
\hline 5 & $38.1 \pm 1.2$ & $34.0 \pm 1.5$ & $31.0 \pm 0.7$ & 33 & $41.3 \pm 2.6$ & $37.3 \pm 0.9$ & $23.9 \pm 0.8$ & 61 & $27.9 \pm 1.6$ & $32.0 \pm 0.9$ & $33.0 \pm 2.3$ & 89 & $31.0 \pm 0.3$ & $26.7 \pm 0.3$ & $22.1 \pm 1.0$ \\
\hline 6 & $15.2 \pm 3.2$ & $17.1 \pm 0.3$ & $18.8 \pm 0.7$ & 34 & $31.5 \pm 0.8$ & $33.8 \pm 0.5$ & $33.8 \pm 1.0$ & 62 & $27.6 \pm 0.2$ & $36.7 \pm 0.6$ & $33.9 \pm 1.8$ & 90 & $42.6 \pm 3$. & $29.5 \pm 4.1$ & $25.6 \pm 1.6$ \\
\hline 7 & $30.2 \pm 5$. & $27.0 \pm 3.7$ & $19.5 \pm 0.3$ & 35 & $33.2 \pm 1.8$ & $33.8 \pm 0.5$ & $31.5 \pm 0.2$ & 63 & $28.0 \pm 4.8$ & $25.4 \pm 1.4$ & $29.9 \pm 1.5$ & 91 & $39.3 \pm 1$ & $38.8 \pm 0.4$ & $28.8 \pm 1.2$ \\
\hline 8 & $31.6 \pm 2.1$ & $26.7 \pm 1.3$ & $20.6 \pm 0.4$ & 36 & $41.4 \pm 1.0$ & $37.4 \pm 0.9$ & $29.1 \pm 0.7$ & 64 & $34.8 \pm 6.7$ & $31.5 \pm 1.3$ & $23.8 \pm 0.8$ & 92 & $31.8 \pm 1.6$ & $33.8 \pm 0.6$ & $31.5 \pm 1.7$ \\
\hline 9 & $33.8 \pm 4.7$ & $31.4 \pm 0.3$ & $21.4 \pm 0.7$ & 37 & $22.1 \pm 0.9$ & $26.9 \pm 1.2$ & $37.5 \pm 0.6$ & 65 & $21.5 \pm 5.8$ & $27.1 \pm 0.4$ & $17.3 \pm 1.1$ & 93 & $30.6 \pm 4.1$ & $35.9 \pm 0.6$ & $34.3 \pm 1.0$ \\
\hline 10 & $27.1 \pm 3.8$ & $35.3 \pm 0.8$ & $31.2 \pm 0.8$ & 38 & $30.0 \pm 0.6$ & $31.8 \pm 0.8$ & $31.6 \pm 1.7$ & 66 & $1.6 \pm 1.0$ & $6.3 \pm 0.2$ & $6.1 \pm 0.2$ & 94 & $44.9 \pm 0$. & $20.4 \pm 0.3$ & $27.9 \pm 1.2$ \\
\hline 11 & $40.2 \pm 0.6$ & $38.2 \pm 0.4$ & $25.3 \pm 1.9$ & 39 & $26.7 \pm 3.3$ & $23.1 \pm 5.3$ & $18.7 \pm 1.0$ & 67 & $21.8 \pm 3.8$ & $25.5 \pm 0.2$ & $21.0 \pm 1.4$ & 95 & $22.2 \pm 0.4$ & $27.5 \pm 0.9$ & $35.0 \pm 1.0$ \\
\hline 12 & $24.1 \pm 4.4$ & $22.7 \pm 2.1$ & $20.0 \pm 2.1$ & 40 & $43.1 \pm 2.7$ & $37.9 \pm 1.2$ & $27.3 \pm 1.4$ & 68 & $23.1 \pm 0.8$ & $19.9 \pm 1.2$ & $20.6 \pm 0.8$ & 96 & $28.0 \pm 1.4$ & $30.7 \pm 0.8$ & $30.9 \pm 0.1$ \\
\hline 13 & $19.0 \pm 3$. & $21.4 \pm 4.4$ & $20.6 \pm 0.7$ & 41 & $26.5 \pm 1.2$ & $32.5 \pm 0.8$ & $34.3 \pm 0.4$ & 69 & $32.7 \pm 2.6$ & $29.3 \pm 1.6$ & $20.9 \pm 2.6$ & 97 & $36.6 \pm$ & $36.8 \pm 2.0$ & $27.2 \pm 2.7$ \\
\hline 14 & $36.4 \pm 7.2$ & $37 \pm 2.4$ & $23.2 \pm 1.8$ & 42 & $38.3 \pm 0$ & $27.8 \pm 0.3$ & $28.6 \pm 1.2$ & 70 & $31.2 \pm 3.6$ & $29.7 \pm 0.6$ & $25.1 \pm 0.8$ & 98 & $31.6 \pm 5.2$ & $31.2 \pm 1.0$ & $22.3 \pm 2.8$ \\
\hline 15 & $29.3 \pm 3.9$ & $22.9 \pm 2.7$ & $18.8 \pm 0.7$ & 43 & $25.1 \pm 3.5$ & $18.1 \pm 3.3$ & $17.5 \pm 0.1$ & 71 & $28.2 \pm 2.1$ & $28.5 \pm 4.1$ & $13.9 \pm 0.7$ & 99 & $45.9 \pm 5.9$ & $40.0 \pm 0.3$ & $27.6 \pm 1.0$ \\
\hline 16 & $48.4 \pm 6.1$ & $38.3 \pm 0.9$ & $29.5 \pm 3.1$ & 44 & $20.5 \pm 3.1$ & $17.6 \pm 4.4$ & $15.9 \pm 0.9$ & 72 & $20.4 \pm 2.1$ & $22.2 \pm 0.4$ & $20.5 \pm 1.7$ & 100 & $29.6 \pm 3.1$ & $24.5 \pm 4.2$ & $19.5 \pm 0.8$ \\
\hline 17 & $37.6 \pm 1.5$ & $38 \pm 0.7$ & $27.3 \pm 1.3$ & 45 & $27.3 \pm 1.7$ & $23.8 \pm 0.8$ & $35.0 \pm 1.2$ & 73 & $20.6 \pm 3.3$ & $21.3 \pm 3.3$ & $18.1 \pm 0.8$ & 101 & $39.2 \pm 3.1$ & $37.8 \pm 0.1$ & $25.6 \pm 1.1$ \\
\hline 18 & $40.1 \pm 2.8$ & $38.2 \pm 0.2$ & $26.9 \pm 4.9$ & 46 & $29.5 \pm 2.6$ & $33.4 \pm 1$ & $30.8 \pm 2.2$ & 74 & $24.7 \pm 6.2$ & $27.2 \pm 0.3$ & $21.9 \pm 2.2$ & 102 & $44.7 \pm 4.4$ & $32.1 \pm 11$ & $27.3 \pm 0.8$ \\
\hline 19 & $39.1 \pm 0.7$ & $38.2 \pm 0.5$ & $25.9 \pm 2.3$ & 47 & $29.5 \pm 1.4$ & $35.9 \pm 0.6$ & $31.1 \pm 0.6$ & 75 & $18.9 \pm 3.2$ & $22.4 \pm 0.1$ & $20.0 \pm 1.0$ & 103 & $42.3 \pm 5.9$ & $31.2 \pm 2.2$ & $27.6 \pm 2.8$ \\
\hline 20 & $21.6 \pm 1.3$ & $30.2 \pm 0.6$ & $35.0 \pm 0.7$ & 48 & $15.1 \pm 1.2$ & $20.8 \pm 1.3$ & $37.3 \pm 0.6$ & 76 & $29.9 \pm 2.0$ & $26.3 \pm 2.1$ & $19.8 \pm 0.4$ & 104 & $22.1 \pm 1.2$ & $24.6 \pm 1.1$ & $17.1 \pm 0.9$ \\
\hline 21 & $29.8 \pm 2.9$ & $21.8 \pm 5.3$ & $26.9 \pm 0.8$ & 49 & $36.2 \pm 1.9$ & $35.2 \pm 0.4$ & $29.3 \pm 1.3$ & 77 & $18.8 \pm 5.9$ & $15.9 \pm 1.1$ & $24.0 \pm 0.4$ & 105 & $45.0 \pm 6.1$ & $39.2 \pm 1.1$ & $28.1 \pm 2.4$ \\
\hline 22 & $33.5 \pm 1.7$ & $33.2 \pm 0.4$ & $28.6 \pm 3.0$ & 50 & $23.4 \pm 0.6$ & $33.8 \pm 1.0$ & $31.8 \pm 2.1$ & 78 & $21.1 \pm 1.8$ & $18.9 \pm 2.5$ & $20.7 \pm 2.6$ & 106 & $28.8 \pm 2.9$ & $25.1 \pm 2$ & $24.5 \pm 1.2$ \\
\hline 23 & $28.8 \pm 1.4$ & $32.5 \pm 0.2$ & $33.0 \pm 1.0$ & 51 & $32.2 \pm 2.7$ & $36.5 \pm 0.6$ & $30.4 \pm 0.5$ & 79 & $29.1 \pm 3.0$ & $28.7 \pm 1.2$ & $21.4 \pm 0.6$ & 107 & $32.1 \pm 1.6$ & $29.0 \pm 1.0$ & $23.5 \pm 0.9$ \\
\hline 24 & $35.6 \pm 1.5$ & $36.8 \pm 0.6$ & $28.2 \pm 2.4$ & 52 & $27.9 \pm 0.7$ & $35.5 \pm 0.7$ & $31.0 \pm 1.8$ & 80 & $40.2 \pm 3.5$ & $36.2 \pm 1.3$ & $27.0 \pm 1.4$ & 108 & $38.6 \pm 4.6$ & $37.7 \pm 0.3$ & $20.6 \pm 0.6$ \\
\hline 25 & $41.8 \pm 2.7$ & $38.7 \pm 0.1$ & $25.0 \pm 5.3$ & 53 & $27.3 \pm 1.8$ & $32.4 \pm 1.4$ & $31.3 \pm 0.9$ & 81 & $27.3 \pm 2.6$ & $28.3 \pm 0.4$ & $21.0 \pm 1.7$ & 109 & $36.9 \pm 1.2$ & $33.7 \pm 1.4$ & $22.3 \pm 2.4$ \\
\hline 26 & $28.1 \pm 2.3$ & $31.2 \pm 1.9$ & $36.3 \pm 0.8$ & 54 & $34.8 \pm 1.1$ & $34.1 \pm 2.4$ & $32.8 \pm 0.3$ & 82 & $29.0 \pm 4.9$ & $27.3 \pm 1.5$ & $20.8 \pm 1.4$ & 110 & $36.9 \pm 6.2$ & $30.6 \pm 1.9$ & $27.7 \pm 1.9$ \\
\hline 27 & $24.4 \pm 0.7$ & $33.3 \pm 0.4$ & $35.2 \pm 1.4$ & 55 & $36.5 \pm 3.5$ & $37.3 \pm 0.4$ & $29.9 \pm 1.3$ & 83 & $24.7 \pm 4.0$ & $26.3 \pm 2.6$ & $21.7 \pm 1.8$ & 111 & $32.4 \pm 3.5$ & $36.4 \pm 0.4$ & $31.8 \pm 1.2$ \\
\hline 28 & $20.1+1.0$ & $32.6 \pm 0.5$ & $31.6 \pm 0.8$ & 56 & $20.7 \pm 2.8$ & $31.5 \pm 1.8$ & $34.3 \pm 1.2$ & 84 & $15.8 \pm 3.0$ & $25.9 \pm 1.4$ & $18.1 \pm 0.9$ & 112 & $31.2 \pm 0.9$ & $30.1 \pm 0.4$ & $23.8 \pm 1.5$ \\
\hline
\end{tabular}

\footnotetext{
z) $\mu \mathrm{g}$ Trolox $\mathrm{mg}^{-1} ;{ }^{\mathrm{y})} \mu \mathrm{g} \mathrm{ASC} \mathrm{mg}{ }^{-1} ;{ }^{\mathrm{x})} \mu \mathrm{g} \mathrm{GAE} \mathrm{mg}{ }^{-1}$
} 
$\sim 60^{\circ} \mathrm{N}$ latitude were distributed between 25 and $45 \mu \mathrm{g}$ Trolox $\mathrm{mg}^{-1}$, while $80 \%$ of accessions from $0^{\circ} \sim 30^{\circ} \mathrm{N}$ latitude were assembled in the range of $15 \sim 35 \mu \mathrm{g}$ Trolox $\mathrm{mg}^{-1}$. DPPH value was greater than $40 \mu \mathrm{g}$ ASC $\mathrm{mg}^{-1}$ for one accession from $30^{\circ} \sim 60^{\circ} \mathrm{N}$ latitude and less

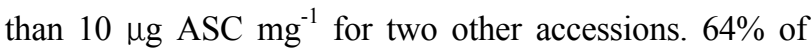
accessions from $30^{\circ} \sim 60^{\circ} \mathrm{N}$ latitude were clustered in 30

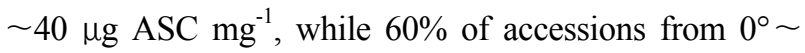
$30^{\circ} \mathrm{N}$ latitude were distributed from 20 to $30 \mu \mathrm{g}$ ASC $\mathrm{mg}^{-1}$. The total polyphenol content showed normal distribution with $90 \%$ of the accessions having values

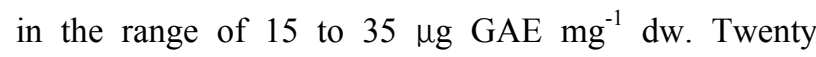
accessions from $30^{\circ} \sim 60^{\circ} \mathrm{N}$ latitude were distributed

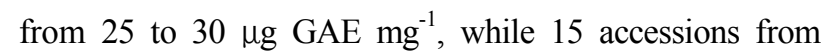
$0^{\circ} \sim 30^{\circ} \mathrm{N}$ and $0^{\circ} \sim 30^{\circ} \mathrm{S}$ were assembled in $20 \sim 25 \mathrm{GAE}$ $\mathrm{mg}^{-1}$ and 30 35 GAE $\mathrm{mg}^{-1}$, respectively (Fig. 4). These results suggested that ABTS, DPPH antioxidant activities and polyphenol content in tomato leaves and stems are

Table 3. Mean, standard deviation and range of antioxidant activity and polyphenol content classified by collection latitudes in stems and leaves extract of 112 tomato germplasm.

\begin{tabular}{ccccccc}
\hline \hline \multirow{2}{*}{ Latitude } & \multicolumn{2}{c}{ ABTS $\left(\right.$ ug Trolox $\left.\mathrm{mg}^{-1}\right)$} & \multicolumn{2}{c}{ DPPH $($ ug ASC mg } \\
\cline { 2 - 7 } & Mean \pm SD & Range & Mean \pm SD & Range & \multicolumn{2}{c}{ Molyphenol $\left(\right.$ ug GAE $\left.\mathrm{mg}^{-1}\right)$} \\
\hline $30^{\circ} \sim 60^{\circ} \mathrm{N}(\mathrm{n}=50)$ & $33.4 \pm 7.4 \mathrm{a}^{\mathrm{z})}$ & $14.1 \sim 48.4$ & $31.6 \pm 6.8 \mathrm{a}$ & $7.3 \sim 40.0$ & $26.8 \pm 5.9 \mathrm{~b}$ & $7.8 \sim 38.9$ \\
$0^{\circ} \sim 30^{\circ} \mathrm{N}(\mathrm{n}=40)$ & $27.3 \pm 8.7 \mathrm{~b}$ & $1.6 \sim 44.9$ & $26.4 \pm 6.4 \mathrm{~b}$ & $6.3 \sim 38.8$ & $23.2 \pm 6.2 \mathrm{c}$ & $6.1 \sim 38.4$ \\
$0^{\circ} \sim 30^{\circ} \mathrm{S}(\mathrm{n}=22)$ & $27.7 \pm 5.7 \mathrm{~b}$ & $15.1 \sim 38.1$ & $30.5 \pm 6.2 \mathrm{a}$ & $17.6 \sim 37.3$ & $30.5 \pm 5.2 \mathrm{a}$ & $15.9 \sim 37.3$ \\
\hline
\end{tabular}

${ }^{\mathrm{z})}$ same letter in each column are not significantly different by duncan's multiple range test, $\mathrm{p}<0.05$

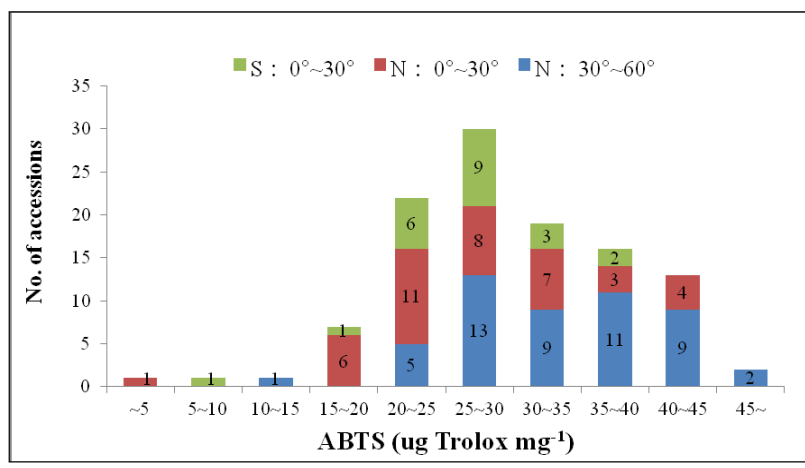

Fig. 2. Distribution of ABTS antioxidant activities in stem and leaf extracts of 112 tomato accessions.

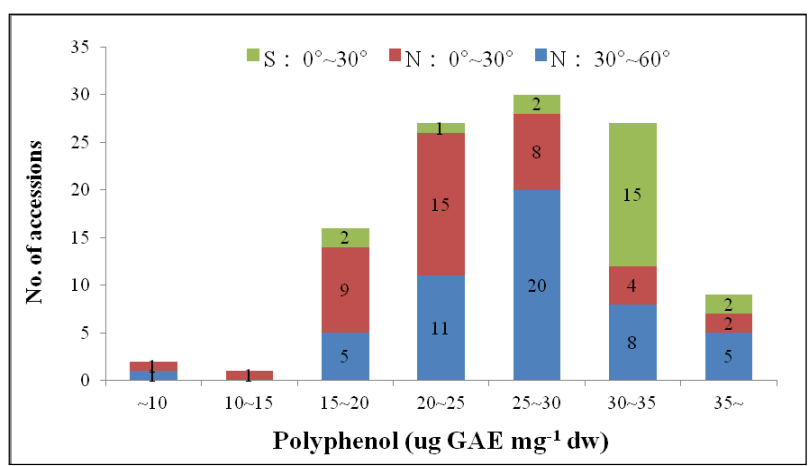

Fig. 4. Distribution of polyphenol contents of stem and leaf extracts of 112 tomato accessions.

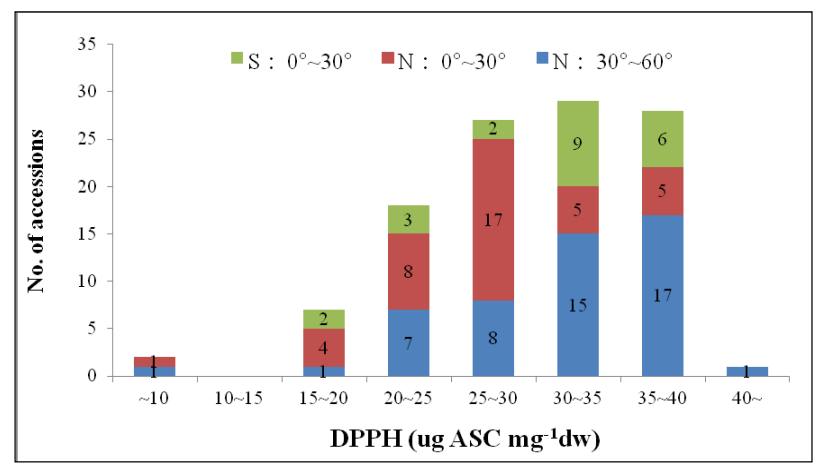

Fig. 3. Distribution of DPPH antioxidant activities in stem and leaf extracts of 112 tomato accessions.

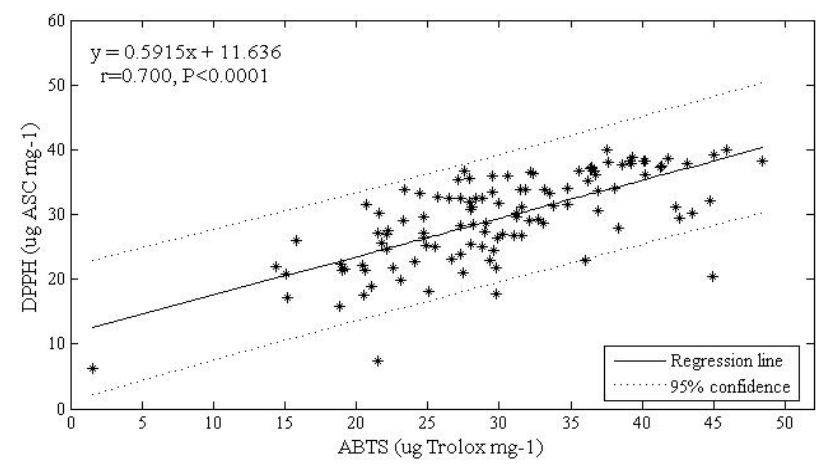

Fig. 5. Relationship between DPPH and ABTS antioxidant activities in stem and leaf extracts of 112 tomato accessions. 
influenced by collection latitudes.

ABTS values showed a significant positive correlation $\left(\mathrm{r}=0.700^{* *}\right)$ with DPPH activity in the 112 tomato germplasm stem and leaf extracts; only three observations fell outside the $95 \%$ confidence interval (dotted lines) (Fig. 5). From the results, IT100506 (KOR) and 702959 (UKR) were recommended as potential sources of natural antioxidants for use in food or feed additives due to their highest antioxidant activity among accessions.

\section{DISCUSSION}

Tomato contains antioxidant, anti-allergic, anti-inflammatory, and anti-bacterial activities (Hanson et al. 2004). The presence of polyphenol might contribute to protective properties in tomato stems and leaves. Phenolics are important mainly due to their function in scavenging free radicals in the human body (Islam et al. 2003). The Folin-Ciocalteau method is commonly used to determine the total polyphenol contents of various samples; gallic acid is typically used as the standard. The color of FolinCiocalteau reagent changes from yellow to blue upon detection of phenolics in an extract due to the chemical reduction of the tungsten and molybdenum oxides mixture in the reagent (Waterhouse 2002). The stable ABTS and DPPH radicals provide the bases of methods of evaluating the free radical scavenging ability of antioxidant substances (Nabavi et al. 2009). In our study, ABTS, DPPH antioxidant activity and polyphenol contents in stem and leaf extracts of the tomato accessions showed wide variations ranging from $1.6 \pm 1.0$ to $48.4 \pm 6.1 \mu \mathrm{g}$ Trolox $\mathrm{mg}^{-1} \mathrm{dw}, 6.3$ \pm 0.2 to $40.0 \pm 0.3 \mu \mathrm{g} \mathrm{ASC} \mathrm{mg}^{-1} \mathrm{dw}$, and $6.1 \pm 0.2$ to $38.9 \pm 0.7 \mu \mathrm{g} \mathrm{GAE} \mathrm{mg}^{-1} \mathrm{dw}$, respectively. As the result, IT100506 (KOR) and 702959 (UKR) were recommended as potential sources of natural antioxidants for use in food or feed additives due to their highest antioxidant activity among accessions (Table 2). The antioxidant capacity in plants was found to be influenced by genotypes, environmental conditions, use of production techniques and storage conditions after post harvesting (Dumas et al. 2003; Kacharava et al. 2009). Northern latitudes have been reported to increase the amounts of phenolics in berries and walnuts (Åkerstöm et al. 2010; Ghasemi et al. 2011). Also in the present study, the ABTS, DPPH antioxidant activities and total polyphenol content in accessions from $30^{\circ} \sim 60^{\circ} \mathrm{N}$ latitude were significantly higher $(\mathrm{P}<0.05)$ than those from $0^{\circ} \sim 30^{\circ} \mathrm{N}$ latitude (Table 3). It is considered that low latitude or high temperature of the geographical origins may lead to the low antioxidant activity and phenolic compounds accumulation. These results were in conformity with other findings that temperature of plant collecting place showed negative correlation with antioxidant activities and total phenolic content (Ghasemi et al. 2011). Pasko et al. (2009) reported a strong positive correlation between ABTS and DPPH antioxidant activity in amaranth and quinoa seeds. Similar result was found in the current study. ABTS values showed a significant positive correlation $\left(\mathrm{r}=0.700^{* *}\right)$ with DPPH activity in the stem and leaf extracts of 112 tomato germplasm (Fig. 5). This study will provide valuable information for tomato breeders and growers in developing and producing functional food or feed additives resources.

\section{ACKNOWLEDGMENT}

This work was supported by the Rural Development Administration (RDA), Republic of Korea (Project No. PJ008625) and postdoctoral program.

\section{REFERENCES}

Åkerstöm A, Jaakola L, Bång U, Jäderlund A. 2010. Effects of latitude-related factors and geographical origin on anthocyanidin concentrations in fruits of Vaccinium myrtillus L. Agric. Food Chem. 58: 11939-11945.

Arnao MB. 2000. Some methodological problems in the determination of antioxidant activity using chromogen radicals: A practical case. Trends Food Sci. Technol. 11: 419-421.

Barceloux DG. 2009. Potatoes, tomatoes, and solanine toxicity (Solanum tuberosum L., Solanum lycopersicum L.). Dis.-a-Month. 55: 391-402.

Block G, Langseth L. 1994. Antioxidant vitamins and disease 
prevention. Food Technol. 48: 80-84.

Borguini R, Torres E. 2009. Tomatoes and tomato products as dietary sources of antioxidants. Food Rev. Intern. 25: 313-325.

Dumas Y, Dadomo M, Di Lucca G, Grolier P. 2003. Effects of environmental factors and agricultural techniques on antioxidant content of tomatoes. J. Sci. Food Agric. 83: 369-382.

Ghasemi K, Ghasemi Y, Ehteshamnia A, Nabavi SM, Nabavi SF, Ebrahimzadeh MA, Pourmorad F. 2011. Influence of environmental factors on antioxidant activity, phenol and flavonoids contents of walnut (Juglans regia L.) green husks. J. Med. Plant Res. 5: 1128-1133.

Hanson PM, Yang R, Wu J, Chen J, Ledesma D, Tsou CS. 2004. Variation for antioxidant activity and antioxidants in tomato. J. Am. Soc. Hortic. Sci. 129: 704-711.

Islam MS, Yoshimoto M, Ishigure K, Okuno S, Yamakawa O. 2003. Effect of artificial shading and temperature on radical scavenging activity and polyphenolic composition in sweetpotato (Ipomoea batatas L.) leaves. J. Am. Soc. Hortic. Sci. 128: 182-187.

Jung EJ, Bae MS, Jo EK, Jo YH, Lee SC. 2011. Antioxidant activity of different parts of eggplant. J. Med. Plant Res. 5: 4610-4615.

Kacharava N, Chanishvili S, Badridze G, Chkhubianishvili E, Janukashvili N. 2009. Effect of seed irradiation on the content of antioxidants in leaves of Kidney bean, Cabbage and Beet cultivars. Aust. J. Crop Sci. 3: 137-145.

Kotkov Z, Hejtmnkov A, Lachman J. 2009. Determination of the influence of variety and level of maturity of the content and development of carotenoids in tomatoes. Czech J. Food Sci. 27: 200-203.

Lee DJ, Lee JY. 2004. Antioxidant activity by DPPH assay. Korean J. of Crop Sci. 49: 187-194.
Mcgee H. 2009. Accused, yes, but probably not a killer. The New York Times. pp. D1

Munir A, Sultana B, Babar T, Bashir A, Amjad M. Hassan Q. 2012. Investigation on the antioxidant activity of leaves, fruit and stem bark of Dhraik (Melia azedarach). Eur. J. Appl. Sci. 4: 47-51.

Nabavi SM, Ebrahimzadeh MA, Nabavi SF, Fazelian M, Eslami B. 2009. In vitro antioxidant and free radical scavenging activity of Diospyros lotus and Pyrus boissieriana growing in Iran. Phcog. Mag. 5: 122-126.

Pasko P, Bartoń H, Zagrodzki P, Gorinstein S, Folta M, Zachwieja Z. 2009. Anthocyanins, total polyphenols and antioxidant activity in amaranth and quinoa seeds and sprouts during their growth. Food Chem. 15: 994-998.

Pinela J, Barros L, Carvalho AM, Ferreira IC. 2012. Nutritional composition and antioxidant activity of four tomato (Lycopersicon esculentum L.) farmer varieties in Northeastern Portugal homegardens. Food Chem. Toxicol. 50: 829-834.

Re R, Pellegrini N, Proteggente A, Pannala A, Yang M, Rice-Evans C. 1999. Antioxidant activity applying an improved ABTS radical cationdecolorisation assay. Free Rad. Biol. Med. 26: 1231-1237.

Truong VD, Mcfeeters RF, Thompson RT, Dean LL, Shofran B. 2007. Phenolic acid content and composition in leaves and roots of common commercial sweet potato (Ipomea batatas L.) cultivars in the United States. J. Food Sci. 72: 343-349.

Waterhouse AL. 2002. Determination of total phenolics. In current protocols in food analytical chemistry. I1.1. 1-I1.1.7. John Wiley \& Sons Inc. New York.

Zornoza P, Esteban RM. 1984. Flavonoids content of tomato plants for the study of the nutritional status. Plant Soil 82: 269-271. 